\section{Twin study of symptom dimensions in psychoses}

\author{
ALASTAIR G. CARDNO, PAK C. SHAM, ROBIN M. MURRAY \\ and PETER McGUFFIN
}

\begin{abstract}
Background Symptomatology
in psychoses can be summarised as quantitative symptom dimensions, but their genetic basis is unknown.
\end{abstract}

Aims To investigate whether genes make an important contribution to symptom dimensions.

Method A total of 224 probandwise twin pairs (106 monozygotic, II8 samegender dizygotic) where probands had psychosis were ascertained from the Maudsley Twin Register in London. Factor analysis was performed on lifetime symptoms rated on the Operational Checklist for Psychotic Disorders (OPCRIT). Correlations of dimension scores within monozygotic and dizygotic pairs concordant for Research Diagnostic Criteria psychoses were performed. Relationships between dimension scores and genetic loading for psychoses were assessed using logistic regression.

Results Patterns offamilial aggregation consistent with a genetic effect were found for the disorganised dimension and for some measures of the negative, manic and general psychotic dimensions. Disorganised dimension scores were related significantly to genetic loading for psychoses.

\section{Conclusions The disorganised} dimension, and possibly other symptom dimensions, may be useful phenotypes for molecular genetic studies of psychoses.

Declaration of interest Support from the Medical Research Council, the National Institute of Mental Health and the Stanley Foundation.
It is well established that symptomatology in psychotic disorders can be ascribed by factor analysis to three main psychotic symptom dimensions (positive, negative and disorganised; Liddle, 1987; Andreasen et al, 1995) and two main mood dimensions (manic and depressive; Lindenmayer et al, 1994; van Os et al, 1997). In addition, studies that include a wide range of positive symptoms often find subdivisions of the positive dimension, e.g. first-rank delusions and paranoid dimensions (Cardno et al, 1997). It is not known whether genetic factors make an important contribution to variation in these symptom dimensions. Studies of affected sib-pairs show modest familial aggregation (Burke et al, 1996; Kendler et al, 1997; Loftus et al, 1998; Cardno et al, 1999a) and familial risk of psychoses has been associated with proband scores on the negative, disorganised, manic and depressive dimensions (Cardno et al, 1997; van Os et al, 1997). In this study, we extended such investigations to a sample of twins with a range of psychoses: we investigated the degree of familial aggregation of dimensions in monozygotic (MZ) and dizygotic (DZ) twin pairs concordant for psychoses; and we investigated whether dimensions are markers of the degree of genetic loading (or liability) for psychoses, by determining whether dimension scores in ill probands predict the presence of psychoses in their co-twins, and whether this effect is greater in MZ than $\mathrm{DZ}$ pairs. If the genes that influence a symptom dimension do not affect the risk of developing psychoses but only influence the clinical presentation (i.e. are modifying genes), there should be greater familial aggregation for the dimension in MZ than in $\mathrm{DZ}$ concordant pairs, but dimension scores in ill twin probands should not predict the presence of psychoses in their co-twins. However, if some genes that affect the risk of developing psychoses also influence what symptoms occur, relevant symptom dimension scores in probands should predict the presence of psychoses in co-twins.

\section{METHOD}

\section{Subjects}

The sample has been described in detail elsewhere (Cardno et al, 1999b). Briefly, probands were ascertained from the Maudsley Twin Register in London as patients of multiple birth who had attended the Maudsley and Bethlem Royal Hospitals between 1948 and 1993, had a same-gender co-twin surviving to 15 years of age and had suffered psychotic symptomatology (Sartorius et al, 1986) or an episode of Research Diagnostic Criteria (RDC) mania or hypomania (Spitzer et al, 1978) at some time in their lives. Thus, a broad concept of psychoses was adopted. Probands were excluded if they had dementia or if psychotic symptoms occurred only during acute organic states.

Zygosity determination was carried out blind to research diagnosis and was based on all available information, including analysis of genetic markers in $42 \%$ of pairs. Agreement between zygosity determination by genetic markers and by resemblance information was $95 \%$.

The sample comprised 224 probandwise pairs (120 male and 104 female) from which 28 probands $(12.5 \%)$ were doubly ascertained; 197 (87.9\%) pairs were White. The mean age of the co-twins at last followup was 46.5 years (s.d.=15.4). There was no substantial ascertainment bias for zygosity (106 MZ and 118 same-gender DZ pairs), but the sample may have contained more severe cases than if we had ascertained via a population-based sample (Cardno et al, 1999b).

\section{Clinical assessment}

This was based on all available clinical information, including a research interview (Gottesman \& Shields' (1972) cued-questions interview) and/or the Schedule for Affective Disorders and Schizophrenia-Lifetime version (SADS-L; Spitzer \& Endicott, 1978 ), in $72.8 \%$ of probands and $59.4 \%$ of co-twins. Interviewed twins gave informed consent to be studied. The RDC lifetimeever diagnoses were made by separate raters for each member of the concordant twin pairs. A non-hierarchical diagnostic approach was employed, that is, twins could qualify for more than one psychotic diagnosis on a lifetime basis. 
Lifetime psychotic and affective symptoms were rated as present/absent using the Operational Criteria Checklist for Psychotic Disorders (OPCRIT; McGuffin et al, 1991). Interrater reliability was assessed on 30 cases and expressed as the mean kappa coefficient $(\kappa)$ between raters for symptoms and the mean Spearman correlation ( $\rho)$ for symptom dimension scores. Variables with a reliability coefficient of less than 0.41 , which is the lower limit of moderate agreement suggested by Landis \& Koch (1977), were excluded from further analysis.

Previous studies have defined dimensions as either symptom scores (Loftus et al, 1998) or factor scores (Burke et al, 1996; Kendler et al, 1997). Because it was not clear which approach would be most useful, both were employed.

Dimensions based on symptom scores were derived from the summation of OPCRIT symptoms that were characteristic of dimensions in previous studies. The following dimensions were included (with their score range, symptoms included and reliability correlations): positive ( $0-2$; delusions, hallucinations; $\rho=0.69)$; negative ( $0-2$; restricted affect, negative formal thought disorder; $\rho=0.65)$; disorganised (0-2; positive formal thought disorder, inappropriate affect; $\rho=0.66)$; manic $(0-5$; mood change, enhanced abilities, decreased need for sleep, speech/thought change, disinhibition; $\rho=0.75)$; depressive $(0-5$; mood change, appetite change, sleep change, negative cognition/suicidal, agitation/ slowness/energy loss; $\rho=0.66$ ); general psychotic (0-6; sum of positive, negative and disorganised dimensions).

\section{Statistical analysis}

Dimensions based on factor scores were derived from two factor analyses of OPCRIT symptoms. The first analysis followed the procedure adopted in our previous factor analyses of OPCRIT symptoms (Cardno et al, 1999a). The 26 individual psychotic symptom items were considered initially in 256 twins rated as having at least one of these symptoms, after excluding twins with only partial clinical information. The symptoms of incoherent speech, blunted affect, delusional perception, thought withdrawal, nihilistic delusions and thought echo were excluded because they were found in fewer than $10 \%$ of twins, and bizarre delusions and other primary delusions were excluded because they had insufficient reliability. This left 18 symptoms for further analysis. A principal components analysis was performed using SPSS Windows Version 6.0 software. The number of substantive factors was determined by the scree test (Cattell, 1966), and these underwent orthogonal (varimax) rotation. Dimension scores then were derived as regression factor scores.

The second factor analysis followed a similar approach but was based on a combination of psychotic and affective symptoms without including an excessively large number of symptoms for analysis. The six psychotic, five manic and five depressive items used to compute the symptom scores were chosen, and all 16 symptoms were included in the analysis, which involved 291 twins.

Spearman correlations were used to assess familial aggregation in twin pairs concordant for psychoses. Correlations were performed for $\mathrm{MZ}$ and $\mathrm{DZ}$ pairs concordant for any psychosis and for $M Z$ pairs concordant for schizophrenia. Too few pairs $(<10)$ were concordant for other diagnoses to allow a meaningful analysis.

Logistic regression analysis was used to analyse associations between symptom dimensions and either familial or genetic loading for psychoses. Pairs where the proband had a particular psychotic diagnosis were selected. Presence/absence of the same diagnosis in co-twins was treated as the dependent variable and the particular dimension as the independent variable, with zygosity and age of co-twins at follow-up as covariates. An effect of a dimension at $P<0.05$ was taken as evidence of an association with familial loading for the psychosis. In such cases a zygosity-by-dimension interaction term was added, and an effect of this term at $P<0.05$ was taken as evidence of an association between the dimension and genetic loading for the psychosis (i.e. a greater effect in $\mathrm{MZ}$ than $\mathrm{DZ}$ pairs). Effects were investigated for lifetime-ever RDC schizophrenia, schizoaffective disorder, mania and all RDC psychoses combined.

\section{RESULTS}

\section{Factor analyses}

In the first factor analysis (of psychotic symptoms), the scree test suggested six substantive factors: disorganised, negative, first-rank delusions, paranoid, other hallucinations and first-rank hallucinations
(Table 1). In the second factor analysis (of both psychotic and affective symptoms) there were three substantive factors: manic, general psychotic and depressive (Table 2).

\section{Within-pair correlations of symptom dimensions}

These are shown in Table 3. In pairs concordant for any psychosis, there were trends towards higher $\mathrm{MZ}$ than $\mathrm{DZ}$ correlations for negative, disorganised, manic and general psychotic symptom scores, and relatively substantial $\mathrm{MZ}$ correlations for these dimensions based on factor scores. An atypical feature of the negative factor was the high loading for inappropriate affect; therefore, the first factor analysis was repeated excluding this symptom. Six similar factors emerged, with the highest loadings on the negative factor for negative formal thought disorder (0.75), catatonia $(0.61)$ and restricted affect $(0.45)$. Withinpair correlations of negative factor scores remained similar (for MZ pairs with any psychosis, $\rho=0.52$ ).

\section{Relationships with genetic loading for psychoses}

The odds ratios (OR) from analysis of relationships between the symptom dimensions and familial loading for psychoses are shown in Table 4. For all psychoses, higher familial loading was associated significantly with higher manic symptom and factor scores and higher disorganised factor scores. In addition, higher paranoid factor scores were associated with lower familial loading for mania. When each of the dimensions showing a significant association with familial loading for all psychoses was included in an interaction term with zygosity (i.e. investigating an association with genetic loading for psychoses), the only significant result was for disorganised factor scores $(\mathrm{OR}=1.47 ; P=0.03)$. The odds ratio for the interaction term remained similar after adjusting for gender $(\mathrm{OR}=$ $1.46 ; P=0.04)$ and age of onset $(\mathrm{OR}=1.42$; $P=0.053)$. Higher disorganised scores are associated with earlier age of onset $(\rho=$ $-0.17 ; P=0.01$ ), so the two variables are not entirely independent, but disorganised scores are better markers of genetic loading for psychoses because age of onset is not associated significantly with genetic loading for psychoses in this sample (interaction $\mathrm{OR}=0.97 ; P=0.44$ ). 
Table I First factor analysis: OPCRIT psychotic symptoms (loadings of $>0.50$ in bold)

\begin{tabular}{|c|c|c|c|c|c|c|}
\hline & Factor I & Factor 2 & Factor 3 & Factor 4 & Factor 5 & Factor 6 \\
\hline Bizarre behaviour & 0.36 & 0.36 & 0.03 & 0.06 & 0.25 & 0.20 \\
\hline Catatonia & 0.10 & 0.53 & -0.16 & -0.08 & 0.39 & -0.26 \\
\hline Speech difficult to understand & 0.83 & 0.34 & 0.00 & 0.04 & 0.08 & 0.02 \\
\hline Positive formal thought disorder & 0.81 & 0.24 & 0.00 & 0.00 & -0.01 & 0.14 \\
\hline Negative formal thought disorder & 0.06 & 0.66 & 0.06 & -0.09 & 0.34 & -0.20 \\
\hline Restricted affect & 0.12 & 0.54 & 0.16 & 0.37 & -0.05 & 0.07 \\
\hline Inappropriate affect & 0.27 & 0.63 & 0.17 & 0.12 & 0.00 & 0.18 \\
\hline Persecutory delusions & 0.02 & 0.05 & 0.06 & 0.80 & 0.07 & 0.04 \\
\hline Grandiose delusions & 0.63 & -0.35 & 0.11 & 0.17 & 0.21 & -0.17 \\
\hline Delusions of influence & 0.15 & -0.06 & 0.16 & 0.62 & 0.29 & 0.01 \\
\hline Delusions of passivity & 0.15 & 0.23 & 0.55 & 0.09 & 0.12 & 0.27 \\
\hline Thought insertion & -0.02 & -0.03 & 0.82 & 0.02 & 0.15 & 0.04 \\
\hline Thought broadcast & 0.00 & 0.08 & 0.77 & 0.15 & -0.06 & -0.01 \\
\hline Third-person voices & 0.07 & -0.04 & 0.34 & -0.08 & 0.03 & 0.67 \\
\hline Commentary voices & 0.05 & -0.03 & -0.06 & 0.15 & 0.17 & 0.73 \\
\hline Abusive voices & -0.07 & 0.32 & 0.02 & 0.54 & -0.04 & 0.47 \\
\hline Other auditory hallucinations & 0.16 & 0.24 & 0.10 & 0.29 & 0.64 & 0.10 \\
\hline Other hallucinations & 0.04 & 0.07 & 0.09 & 0.09 & 0.79 & 0.16 \\
\hline Variance & $21.8 \%$ & $11.0 \%$ & $7.5 \%$ & $7.1 \%$ & $6.6 \%$ & $5.5 \%$ \\
\hline Title & Disorganised & Negative & First-rank delusions & Paranoid & $\begin{array}{l}\text { Other } \\
\text { hallucinations }\end{array}$ & $\begin{array}{c}\text { First-rank } \\
\text { hallucinations }\end{array}$ \\
\hline
\end{tabular}

\section{DISCUSSION}

\section{Familial aggregation of symptom dimensions}

\section{Positive dimension}

The results of this study are not consistent with an important genetic contribution to variation in scores on the positive dimension or its subdivisions within psychoses. Where zygosities could be compared directly, MZ correlations were no higher than DZ correlations. Furthermore, the MZ correlations in general were similar to correlations from studies of affected sib-pairs, including those for factor (up to 0.21 ) and symptom scores (0.21) derived from OPCRIT in the same way as for the current study (Cardno et al, 1999a).

Comparison with the results of the sibpair studies is of interest but should be viewed with caution because of methodological differences from the current study. The closest point of comparison is for schizophrenia because the sib-pair studies have been based on schizophrenia plus related disorders in some cases. Although various definitions of schizophrenia have been employed in the sib-pair studies, this is unlikely to affect substantially the comparison with the current study because all of the concordant $\mathrm{MZ}$ twins fulfilled criteria for RDC, DSM-III-R (American Psychiatric Association, 1987) and ICD10 (World Health Organization, 1993) schizophrenia.

\section{Negative dimension}

The correlations for symptom scores in $\mathrm{MZ}$ pairs with schizophrenia and $\mathrm{DZ}$ pairs with any psychosis were close to zero. This was unexpectedly lower than the correlations found in studies of sib-pairs (range $0.09-0.35$ ) and may be an artefact of the small numbers analysed. The correlation for symptom scores in MZ pairs with any psychosis was modest. However, the correlations for factor scores in $\mathrm{MZ}$ pairs with schizophrenia and any psychosis were more substantial, and greater than twice the correlations for sib-pairs using OPCRIT (0.16; Cardno et al, 1999a). The discrepancy between the correlations for symptom and factor scores may be due to the larger number of symptoms and symptom weightings contributing to the factor scores. It is not accounted for by the atypically high loading for inappropriate affect on the negative factor. These results are consistent with a genetic contribution to variation in the negative dimension, at least as defined by factor scores.

\section{Disorganised dimension}

Correlations of both symptom and factor scores for MZ pairs with schizophrenia and any psychosis were substantial and at least twice as great as the correlations for sib-pairs using OPCRIT (symptom scores= 0.25; factor scores $=0.32$; Cardno et al, $1999 a$ ). The symptom score correlation for DZ pairs with any psychosis was less than half that of the MZ correlation and similar to the OPCRIT-derived sib-pair correlation for schizophrenia/schizoaffective disorder (Cardno et al, 1999a). This is consistent with a genetic contribution to variation in the disorganised dimension.

\section{Manic dimension}

There were markedly negative correlations for MZ pairs with schizophrenia. These were unexpectedly lower than sib-pair correlations (factor score $=0.27$ and symptom score $=0.43$; Kendler et al, 1997) and may be an artefact of the small numbers analysed. The same applies to the low correlations for DZ pairs with any psychosis. In contrast, the correlations for $\mathrm{MZ}$ pairs with any psychosis were more substantial and approximately 1.5 -fold higher than the sib-pair correlation for all non-affective psychoses (0.27 and 0.26; Kendler et al, 
Table 2 Second factor analysis: OPCRIT psychotic and affective symptoms (loadings of $>0.50$ in bold)

\begin{tabular}{|c|c|c|c|}
\hline & Factor I & Factor 2 & Factor 3 \\
\hline Delusions & 0.25 & 0.52 & 0.07 \\
\hline Hallucinations & 0.10 & 0.71 & 0.11 \\
\hline Negative formal thought disorder & 0.01 & 0.54 & -0.05 \\
\hline Restricted affect & -0.07 & 0.69 & 0.07 \\
\hline Positive formal thought disorder & 0.22 & 0.58 & -0.10 \\
\hline Inappropriate affect & 0.03 & 0.74 & -0.05 \\
\hline \multicolumn{4}{|l|}{ Depressive } \\
\hline Mood & -0.13 & -0.01 & 0.68 \\
\hline Appetite & 0.08 & -0.12 & 0.71 \\
\hline Sleep & 0.10 & -0.11 & 0.74 \\
\hline Cognition & 0.04 & 0.13 & 0.65 \\
\hline Behaviour & 0.11 & 0.12 & 0.60 \\
\hline \multicolumn{4}{|l|}{ Manic } \\
\hline Mood & 0.79 & 0.21 & -0.02 \\
\hline Abilities & 0.83 & 0.17 & -0.03 \\
\hline Sleep & 0.80 & -0.10 & 0.18 \\
\hline Speech & 0.85 & 0.06 & -0.02 \\
\hline Disinhibition & 0.80 & 0.14 & 0.16 \\
\hline Variance accounted for & $24.5 \%$ & $15.1 \%$ & $13.2 \%$ \\
\hline Title & Manic & General psychotic & Depressive \\
\hline
\end{tabular}

Table 3 Within-pair Spearman correlations of symptom dimensions in concordant twin pairs

\begin{tabular}{|c|c|c|c|c|c|c|}
\hline \multirow[t]{3}{*}{ Dimension } & \multirow{2}{*}{\multicolumn{2}{|c|}{$\frac{\text { RDC schizophrenia }}{M Z}$}} & \multicolumn{4}{|c|}{ All RDC psychoses } \\
\hline & & & \multicolumn{2}{|c|}{$M Z$} & \multicolumn{2}{|c|}{$\mathrm{DZ}$} \\
\hline & $n$ & $\rho$ & $n$ & $\rho$ & $n$ & $\rho$ \\
\hline \multicolumn{7}{|l|}{ Symptom scores } \\
\hline Positive & 13 & - & 44 & 0.19 & 13 & 0.27 \\
\hline Negative & 13 & -0.01 & 44 & 0.27 & 13 & -0.03 \\
\hline Disorganised & 13 & 0.05 & 44 & $0.63^{* * *}$ & 13 & 0.24 \\
\hline Manic & 13 & -0.35 & 44 & $0.42^{*}$ & 13 & -0.07 \\
\hline Depressive & 13 & 0.02 & 44 & 0.21 & 13 & -0.12 \\
\hline General psychotic & 13 & 0.16 & 44 & $0.47 * *$ & 13 & 0.27 \\
\hline \multicolumn{7}{|l|}{ Factor scores (FAI) } \\
\hline First-rank delusions & 13 & -0.29 & 43 & -0.11 & 9 & - \\
\hline First-rank hallucinations & 13 & 0.14 & 43 & 0.10 & 9 & - \\
\hline Paranoid & 13 & -0.03 & 43 & 0.24 & 9 & - \\
\hline Other hallucinations & 13 & 0.20 & 43 & 0.05 & 9 & - \\
\hline Negative & 13 & 0.46 & 43 & $0.59 * * *$ & 9 & - \\
\hline Disorganised & 13 & $0.64 *$ & 43 & $0.42^{*}$ & 9 & - \\
\hline \multicolumn{7}{|l|}{ Factor scores (FA2) } \\
\hline Manic & 13 & -0.46 & 43 & $0.44 * *$ & II & -0.19 \\
\hline Depressive & 13 & -0.23 & 43 & 0.21 & II & 0.26 \\
\hline General psychotic & 13 & 0.04 & 43 & $0.53 * * *$ & II & 0.17 \\
\hline
\end{tabular}

RDC, Research Diagnostic Criteria; MZ, monozygotic; DZ, dizygotic; $n$, number of twin pairs counted pairwise; $\rho$, Spearman correlation coefficient; FAl, first factor analysis; FA2, second factor analysis.

I. Of the $26 \mathrm{MZ}$ twins from pairs concordant for schizophrenia, 25 had the maximum score of 2

2. There were too few concordant $D Z$ pairs from FAI for analysis $(n=9)$.

$* P<0.05 ; * * P<0.005 ; * * * P<0.0005$
1997). These results are consistent with a genetic contribution to variation in manic dimension scores within psychoses.

\section{Depressive dimension}

Correlations were uniformly low. In some cases this could have been an artefact of small samples, but even for MZ pairs with any psychosis the correlation was marginally lower than for sib-pairs with any nonaffective psychosis (0.28; Kendler et al, 1997). These results do not suggest that genetic effects make an important contribution to variation in depressive dimension scores within psychoses. Although major depressive disorder has a heritability of $40 \%$ or higher (Kendler \& Prescott, 1999), there may be differences between this disorder in general and variation of depressive symptomatology in psychoses.

\section{General psychotic dimension}

Correlations were low in $\mathrm{MZ}$ twins with schizophrenia and similar to the correlation in our sib-pair sample (0.12; details available from P.M. upon request). This could be because there is little genetic contribution to variation in this dimension in schizophrenia, but equally it could be an artefact of the small numbers in this part of the sample. In contrast, for all psychoses the MZ correlations were consistently more substantial (0.47 and 0.53 ) and considerably greater than the corresponding DZ correlations ( 0.27 and 0.17$)$. Some caution again is required in interpreting the DZ correlations because of the small numbers, but the pattern is consistent with a genetic contribution to variation in the general psychotic dimension in individuals with any psychosis.

\section{Relationships with genetic loading for psychoses}

Only the disorganised dimension, defined by factor scores, showed a significant association with genetic loading for all psychoses combined. This is partly consistent with previous family history studies. Disorganised scores in probands with RDC schizophrenia have been associated with familial risk of psychosis (Cardno et al, 1997; van Os et al, 1997), but in one study (van Os et al, 1997) negative dimension scores in probands with any psychosis best predicted the familial risk of psychosis. It is also notable that there are similarities between the disorganised dimension and the hebephrenic 
Table 4 Logistic regression of RDC lifetime-ever psychotic diagnoses in co-twins on symptom dimensions in probands, conditional on zygosity and age of co-twins at last follow-up

\begin{tabular}{|c|c|c|c|c|}
\hline \multirow[t]{2}{*}{ Dimension } & \multicolumn{4}{|c|}{ Odds ratios } \\
\hline & $\begin{array}{l}\text { Schizophrenia } \\
\qquad(n=106)\end{array}$ & $\begin{array}{c}\text { Schizoaffective } \\
\qquad(n=45)\end{array}$ & $\begin{array}{l}\text { Mania } \\
(n=49)\end{array}$ & $\begin{array}{c}\text { All psychoses } \\
(n=222)^{\prime}\end{array}$ \\
\hline \multicolumn{5}{|l|}{ Symptom scores } \\
\hline Positive $(0-2)^{2}$ & - & 1.62 & 1.94 & 1.02 \\
\hline Negative (0-2) & 1.09 & 0.10 & 0.12 & 1.06 \\
\hline Disorganised $(0-2)$ & $\mathrm{I} .54$ & 0.87 & 2.52 & 1.23 \\
\hline Manic (0-5) & 0.87 & 1.06 & 1.67 & $1.2 I^{*}$ \\
\hline Depressive (0-5) & 1.01 & 1.57 & 1.23 & 0.99 \\
\hline General psychotic (0-6) & 1.27 & 0.68 & 1.20 & 1.07 \\
\hline \multicolumn{5}{|l|}{ Factor scores (FAI) } \\
\hline First-rank delusions & 0.66 & 0.83 & 4.49 & 0.82 \\
\hline First-rank hallucinations & 0.75 & 1.05 & 1.29 & 0.91 \\
\hline Paranoid & 0.94 & 1.33 & $0.33^{*}$ & 0.74 \\
\hline Other hallucinations & 1.45 & 0.95 & 0.93 & 1.25 \\
\hline Negative & 1.21 & 0.55 & 0.59 & 1.02 \\
\hline Disorganised & 1.48 & 1.26 & 2.57 & $1.62 *$ \\
\hline \multicolumn{5}{|l|}{ Factor scores (FA2) } \\
\hline Manic & 0.75 & 1.18 & 3.54 & $1.45^{*}$ \\
\hline Depressive & 0.98 & 1.88 & 1.27 & 0.97 \\
\hline General psychotic & 1.58 & 0.33 & 1.23 & 1.07 \\
\hline
\end{tabular}

RDC, Research Diagnostic Criteria; FAI, first factor analysis; FA2, second factor analysis.

I. One monozygotic and one dizygotic proband did not qualify for any RDC psychotic diagnosis; and 215 probands had

FAl scores (i.e. had at least one of the symptoms entered into the factor analysis).

2. The odds ratio for positive symptom scores in schizophrenia was not reported because 103/106 probands had the maximum score of two.

$* P<0.05$ (d.f. $=$ I).

schizophrenia subtype, and hebephrenia has been associated with relatively high familial loading for schizophrenia in previous studies of Maudsley and other twin samples (Farmer et al, 1984; McGuffin et al, 1987; Onstad et al, 1991).

The disorganised dimension, defined by symptom scores, showed a trend towards an association with familial loading for all psychoses that failed to reach statistical significance $(\mathrm{OR}=1.23 ; P=0.32)$. This may be because the $0-2$ symptom scale resulted in less statistical power than the continuous factor scores. The pattern of symptom weightings that contributed to disorganised factor scores also potentially could account for the difference. In addition to positive formal thought disorder, the disorganised factor included a high loading for grandiose delusions. This symptom has not been part of the disorganised dimension in most previous factor analytical studies, where only global delusion scores have been considered (Liddle, 1987; Andreasen et al, 1995), but has had moderate or high loadings in previous studies using OPCRIT in subjects with schizophrenia or schizoaffective disorder (Cardno et al, 1999a) and using the Scales for the Assessment of Positive and Negative Symptoms (SAPS/ SANS; Andreasen, 1984a,b) for individual symptoms in subjects with a range of psychoses (Toomey et al, 1997).

The presence of grandiose delusions in the disorganised dimension raises the question of the relationship between disorganised and manic symptoms. Disorganised and manic factor scores were correlated significantly even when grandiose delusions were omitted from the disorganised dimension $(\rho=0.29 ; P<0.0005)$, which is consistent with previous findings (Johnstone $\&$ Frith, 1996). The apparent relationship between disorganised and manic dimensions could be due to mislabelling of symptoms, because it can be difficult to distinguish reliably the knight's-move thinking from a flight of ideas (Andreasen, 1979). It is possible also that the two dimensions comprise distinct symptoms that are associated with common pathological processes; for example, disorganisation has been associated with cognitive disinhibition (Liddle \& Morris, 1991) and disinhibition is an important clinical feature of mania.

The lack of relationship between the positive dimension and its subdivisions with familial loading for psychoses is consistent with previous studies (Kendler et al, 1994; Cardno et al, 1997; van Os et al, 1997). The lack of effect for the general psychotic dimension provides some support for the approach of dividing clinical variation within psychoses into a number of symptom dimensions. The lack of effect for the depressive dimension for all psychoses is consistent with the results of van Os et al (1997), who found an association only in schizoaffective disorder. The lack of effect for the negative dimension is consistent with some studies (Kendler $e t$ al, 1994; Cardno et al, 1997) but not others (Dworkin \& Lenzenweger, 1984; van Os et al, 1997). One of the positive studies found the effect for clinical schizophrenia in $\mathrm{MZ}$ twins (Dworkin \& Lenzenweger, 1984). In the current study there was a trend towards the largest (non-significant) odds ratio with schizophrenia (1.21), so a small effect cannot be excluded. The other family history study (van Os et al, 1997) found the effect for all psychoses using OPCRIT, but the dimensions differed from those in the current study in that they included illness history variables as well as symptoms. The current negative factor included inappropriate affect, which is more commonly associated with disorganisation (and indeed had a moderate loading on this dimension). However, we performed a further analysis of negative factor scores excluding the inappropriate affect, and the results for the relationship between the negative dimension and familial liability to all psychoses $(\mathrm{OR}=1.07 ; \quad P=0.68)$ and schizophrenia $(\mathrm{OR}=1.24 ; P=0.38)$ remained similar. Therefore, the relationship between the negative dimension and familial loading for psychoses remains inconclusive.

\section{Limitations}

As noted above, the twin sample is probably selected for severity, and the small numbers in some categories limited the number of analyses that could be performed reasonably and limited direct MZ-DZ comparison with all psychoses combined. There is also a need for caution when comparing the 
results of the current study with the results of sib-pair studies: there are general methodological differences (e.g. concerning ascertainment); the twin sample includes same-gender pairs only; and there are potential twin versus sibling differences regarding familial aggregation of dimensions. In addition, comparison of factor scores across studies should be done cautiously because of variation in the patterns of factor loadings in different samples. Different results also could have occurred if dimensions were based on other rating scales. However, factor scores derived from OPCRIT and SAPS/SANS in our sib-pair study were correlated strongly (Cardno et al, 1999a).

Because the data were either ordinal or not easily transformed to a normal distribution, we employed non-parametric Spearman correlations. Confidence intervals therefore could not be calculated. It would be possible technically to apply biometric model fitting to make heritability estimates for the dimensions, but the sample size was not sufficient for this to be done with confidence.

The MZ-DZ differences in familial aggregation of dimensions will tend to be underestimated if genetic risk factors for psychotic disorders contribute to the variation in symptom dimensions. This is because pairs of DZ twins who both have psychosis will tend to share susceptibility alleles more frequently than the expected $50 \%$, and so will be more like $\mathrm{MZ}$ twins at loci relevant to the aetiology of psychoses. However, the intrapair correlations in affected sib-pairs are modest, suggesting that genetic effects are due predominantly to genes with modifying effects on illness expression, with susceptibility genes themselves probably having a more minor role.

In the logistic regression analysis, numerous statistical tests were performed and so the result for disorganisation could be a type I error. However, the support from previous studies (Cardno et al, 1997; van Os et al, 1997) argues against this. It seems more likely that some of the nonsignificant findings in the current study were type II errors. For example, the association between manic factor scores and familial loading for mania was non-significant for an odds ratio of 3.54 .

Future genetic studies that include cognitive, physiological and brain imaging data may shed further light on the genetic basis of symptom dimensions and their relationship with both pathological processes

\section{CLINICAL IMPLICATIONS}

- There is consistent evidence for a genetic contribution to variation in the disorganised symptom dimension, and to a lesser extent to the negative, manic and general psychotic dimensions.

Individuals with high disorganised dimension scores have relatively high genetic loading for psychotic disorders.

The disorganised dimension therefore may be a useful phenotype for molecular genetic studies of psychotic disorders.

\section{LIMITATIONS}

Although this is the largest twin study of operationally defined psychotic disorders to date, numbers of concordant pairs in some categories were small.

- Dimensions based on other rating scales could produce different results.

- The sample may have contained more severe cases than if it had been ascertained via a population-based sample.

ALASTAIR G. CARDNO, MRCPsych, Departments of Psychological Medicine and Medical Genetics, University of Wales College of Medicine, Cardiff; PAKC. SHAM, MRCPsych, ROBIN M. MURRAY, FRCPsych, PETER McGUFFIN, FRCPsych, Institute of Psychiatry, London

Correspondence: Professor Peter McGuffin, Social Genetic and Developmental Psychiatry (SGDP) Research Centre, Institute of Psychiatry, De Crespigny Park, London SE5 8AF, UK. Tel: 0207848 087I; Fax: 0207848 0866; e-mail: p.mcguffin@iop.kcl.ac.uk

(First received 2 I September 2000, final revision I3 February 200I, accepted I5 February 200I)

in psychotic disorders and extremes of variation in the general population.

\section{ACKNOWLEDGEMENTS}

This work was carried out while one of the authors (A.G.C.) was a Medical Research Council (UK) Clinical Training Fellow. The National Institute of Mental Health (MH44359) and the Stanley Foundation provided grant support that enabled collection of the clinical data. The authors thank Jane Marshall, Bina Coid, Alison Macdonald, Tracy Ribchester Nadia Davies, Piero Venturi, Lisa Jones, Shôn Lewis, Irving Gottesman, Anne Farmer and Adrianne Reveley for their work on this study.

\section{REFERENCES}

American Psychiatric Association (1987) Diagnostic and Statistical Manual of Mental Disorders (3rd edn, revised) (DSM-III-R). Washington, DC: APA.

Andreasen, N. C. (1979) Thought, language, and communication disorders. I: Clinical assessment, definition of terms, and evaluation of their reliability Archives of General Psychiatry, 36, 1315-1321.

- (1984a) The Scale for the Assessment of Negative Symptoms (SANS). lowa City, IA: University of lowa. (1984b) The Scale for the Assessment of Positive Symptoms (SAPS). lowa City, IA: University of lowa.

_, Arndt, S., Alliger, R., et al (1995) Symptoms of schizophrenia: methods, meanings, and mechanisms. Archives of General Psychiatry, 52, 34I-35I.

Burke, J. G., Murphy, B. M., Bray, J. C., et al (1996) Clinical similarities in siblings with schizophrenia. American Journal of Medical Genetics (Neuropsychiatric Genetics), 67, 239-243.

Cardno, A. G., Holmans, P. A., Harvey, I., et al (1997) Factor-derived subsyndromes of schizophrenia and familial morbid risks. Schizophrenia Research, $\mathbf{2 3}$ $231-238$.

_, Jones, L. A., Murphy, K. C., et al (1999a)

Dimensions of psychosis in affected sibling pairs. Schizophrenia Bulletin, 25, 84I-850.

_ , Marshall, E. J., Coid, B., et al (1999b) Heritability estimates for psychotic disorders: the Maudsley twin psychosis series. Archives of General Psychiatry, 56, 162-168.

Cattell, R. B. (1966) The scree test for the number of factors. Multivariate Behavioral Research, I, 245-276.

Dworkin, R. H. \& Lenzenweger, M. F. (1984) Symptoms and the genetics of schizophrenia: implications for diagnosis. American Journal of Psychiatry, I4I, |54I-I546. 
Farmer, A. E., McGuffin, P. \& Gottesman, I. I. (1984) Searching for the split in schizophrenia: a twin study perspective. Psychiatry Research, 13, 109-118.

Gottesman, I. I. \& Shields, J. (1972) Schizophrenia and Genetics: A Twin Vantage Point. New York: Academic Press.

Johnstone, E. C. \& Frith, C. D. (1996) Validation of three dimensions of schizophrenic symptoms in a large unselected sample of patients. Psychological Medicine, 26, 669-679.

Kendler, K. S. \& Prescott, C. A. (1999) A populationbased twin study of lifetime major depression in men and women. Archives of General Psychiatry, 56, 39-44.

_ , McGuire, M., Gruenberg, A. M., et al (1994)

Clinical heterogeneity in schizophrenia and the pattern of psychopathology in relatives: results from an epidemiologically based family study. Acta Psychiatrica Scandinavica, 89, 294-300.

—, Karkowski-Shuman, L., O'Neill, F. A., et al (1997) Resemblance of psychotic symptoms and syndromes in affected sibling pairs from the Irish study of high-density schizophrenia families: evidence for possible etiologic heterogeneity. American Journal of Psychiatry, 154, 191-198.
Landis, J. R. \& Koch, G. C. (1977) The measurement of observer agreement for categorical data. Biometrics, 33, $1089-109 \mid$.

Liddle, P. F. (1987) The symptoms of chronic schizophrenia. A re-examination of the positivenegative dichotomy. British Journal of Psychiatry, I5I |45-151.

— \& Morris, D. L. (199I) Schizophrenic syndromes and frontal lobe performance. British journal of Psychiatry, I58, 340-345.

Lindenmayer, J-P., Bernstein-Hyman, R.

\& Grochowski, S. (1994) Five-factor mode

of schizophrenia: initial validation. Journal of Nervous and Mental Disease, 182, 631-638.

Loftus, J., DeLisi, L. E. \& Crow, T. J. (1998) Familia associations of subsyndromes of psychosis in affected sibling pairs with schizophrenia and schizoaffective disorder. Psychiatry Research, 80, I0I-III.

McGuffin, P., Farmer, A. \& Gottesman, I. I. (1987) Is there really a split in schizophrenia? The genetic evidence. British Journal of Psychiatry, 150, 58I-592.

_, _ \& Harvey, I. (1991) A polydiagnostic application of operational criteria in studies of psychotic illness: development and reliability of the OPCRIT system. Archives of General Psychiatry, 48, 764-770.
Onstad, S., Skre, I., Torgersen, S., et al (1991) Subtypes of schizophrenia: evidence from a twin-family study. Acta Psychiatrica Scandinavica, 84, 203-206.

Sartorius, N., Jablensky, A., Korten, A., et al (1986) Early manifestations and first-contact incidence of schizophrenia in different cultures. Psychological Medicine, 16, 909-928.

Spitzer, R. L. \& Endicott, J. (1978) Schedule for Affective Disorders and Schizophrenia - Lifetime Version. New York: New York State Psychiatric Institute.

_ , Endicott, J. \& Robins, E. (1978) Research Diagnostic Criteria for a Selected Group of Functional Disorders (3rd edn). New York: New York State Psychiatric Institute.

Toomey, R., Kremen, W. S., Simpson, J. C., et al (1997) Revisiting the factor structure for positive and negative symptoms: evidence from a large heterogeneous group of psychiatric patients. American Journal of Psychiatry, 154, 37I-377.

van Os, J., Marcelis, M., Sham, P., et al (1997) Psychopathological syndromes and familial morbid risk of psychosis. British Journal of Psychiatry, I70, 24I-246.

World Health Organization (1993) The ICD-10 Classification of Mental and Behavioural Disorders. Diagnostic Criteria for Research. Geneva: WHO. 